\title{
Analytical Method for Next-to-Leading-Order QCD Corrections to Double-Higgs Production
}

\author{
Roberto Bonciani, ${ }^{1,2, *}$ Giuseppe Degrassi, ${ }^{3,4, \dagger}$ Pier Paolo Giardino, ${ }^{5, *}$ and Ramona Gröber, ${ }^{6,}$ \\ ${ }^{1}$ Dipartimento di Fisica, Sapienza-Università di Roma, Piazzale Aldo Moro 5, 00185, Rome, Italy \\ ${ }^{2}$ INFN Sezione di Roma, Piazzale Aldo Moro 2, 00185, Rome, Italy \\ ${ }^{3}$ Dipartimento di Matematica e Fisica, Università di Roma Tre, I-00146 Rome, Italy \\ ${ }^{4}$ INFN, Sezione di Roma Tre, 00146 Rome, Italy \\ ${ }^{5}$ Department of Physics, Brookhaven National Laboratory, Upton, New York 11973, USA \\ ${ }^{6}$ Institute for Particle Physics Phenomenology, Department of Physics, Durham University, Durham, DH1 3LE, United Kingdom
}

(Received 13 July 2018; published 19 October 2018)

\begin{abstract}
We propose a new method to calculate analytically higher-order perturbative corrections and we apply it to the calculation of the two-loop virtual corrections to Higgs pair production through gluon fusion. The method is based on the expansion of the amplitudes in terms of a small Higgs transverse momentum. This approach gives a very good approximation (better than per mille) of the partonic cross section in the centerof-mass energy region $\sqrt{\hat{s}} \lesssim 750 \mathrm{GeV}$, where $\sim 95 \%$ of the total hadronic cross section is concentrated. The presented method is general and can be applied in a straightforward way to the computation of virtual higher-order corrections to other $2 \rightarrow 2$ processes, representing an improvement with respect to calculations based on heavy mass expansions.
\end{abstract}

DOI: 10.1103/PhysRevLett.121.162003

Introduction.-The experimental exploration of the properties of the Higgs boson is one of the major targets of the Large Hadron Collider (LHC). However the self-couplings of the Higgs boson, which in the standard model are fully determined in terms of the mass of the Higgs boson and the Fermi constant, have not been probed yet. While the quartic Higgs self-coupling is not directly accessible at the LHC $[1,2]$, the trilinear self-coupling might be measurable from Higgs pair production processes [3-17].

Those processes, in particular Higgs pair production in gluon fusion, are also sensitive to new physics, that can greatly modify their rates [18-22]. Bounds on $g g \rightarrow H H$ for different final states are reported in Refs. [23-28].

Therefore, a precise prediction of the gluon fusion channel is essential to determine the Higgs trilinear selfcoupling and constrain new physics. At leading order (LO) the gluon fusion process has been known since the 1980s [29]. At next-to-leading order (NLO) this process is fully known only numerically [30,31], while analytical results are available in the heavy top mass $\left(m_{t}\right)$ limit [32-34] and partially in the light $m_{t}$ limit [35]. In Ref. [36] a method was proposed for obtaining an analytical result combining large top mass expansion and a threshold expansion by means of Padé approximants.

Published by the American Physical Society under the terms of the Creative Commons Attribution 4.0 International license. Further distribution of this work must maintain attribution to the author(s) and the published article's title, journal citation, and DOI. Funded by SCOAP ${ }^{3}$.
The limits of Refs. [32-35] well describe the Higgs pair production in the regions $\sqrt{\hat{s}}<300 \mathrm{GeV}$ and $\sqrt{\hat{s}}>750 \mathrm{GeV}$, respectively, where $\sqrt{\hat{s}}$ is the partonic center-of-mass energy, but fail to describe the intermediate region.

We propose a new approach for the analytical calculation of the virtual NLO corrections to the Higgs pair production through gluon fusion. The method is based on the expansion of the amplitudes around a small Higgs transverse momentum $p_{T}$ and Higgs mass $m_{h}$. After properly expanding, the resulting amplitudes are functions of only $m_{t}$ and $\sqrt{\hat{s}}$ and can be calculated analytically without resorting to further expansions. With this method we are able to correctly describe the Higgs pair production in the region $\sqrt{\hat{s}} \lesssim 750 \mathrm{GeV}$, nicely complementing the present literature. It must also be noted that, due to the shape of the gluon parton distribution functions, this region represents $95 \%$ of the total hadronic cross section.

Our approach has the virtue of covering larger regions of the phase space with respect to approaches based on heavy mass expansions or high energy expansions and can be easily implemented to other $2 \rightarrow 2$ processes.

In this Letter we describe the basics of the method, and the main results of our calculation, while we will reserve a more detailed discussion of the computation to future works.

Notation and definitions.-In this section we introduce the notation we will use in the rest of the Letter and define a set of kinematical variables. The amplitude $g_{a}^{\mu}\left(p_{1}\right) g_{b}^{\nu}\left(p_{2}\right) \rightarrow H\left(p_{3}\right) H\left(p_{4}\right)$ can be written as 


$$
A^{\mu \nu}=\frac{G_{\mu}}{\sqrt{2}} \frac{\alpha_{s}\left(\mu_{R}\right)}{2 \pi} \delta_{a b} T_{F} \hat{S}\left[A_{1}^{\mu \nu} F_{1}+A_{2}^{\mu \nu} F_{2}\right],
$$

where $G_{\mu}$ is the Fermi constant, $\alpha_{s}\left(\mu_{R}\right)$ is the strong coupling defined at the renormalization scale $\mu_{R}$, and $T_{F}=$ $1 / 2$ is the normalization factor for the fundamental representation of $S U\left(N_{c}\right)$. In Eq. (1) $A_{1,2}^{\mu \nu}$ are the orthogonal projectors onto the spin- 0 and spin-2 states, respectively, while the corresponding form factors $F_{1,2}$ are functions of $m_{t}, m_{h}$, and the partonic Mandelstam variables (all momenta are assumed incoming)

$\hat{s}=\left(p_{1}+p_{2}\right)^{2}, \quad \hat{t}=\left(p_{1}+p_{3}\right)^{2}, \quad \hat{u}=\left(p_{2}+p_{3}\right)^{2}$,

via

$$
F_{1}=F_{1}\left(\hat{s}, \hat{u}, m_{t}^{2}, m_{h}^{2}\right), \quad F_{2}=F_{2}\left(\hat{s}, \hat{u}, m_{t}^{2}, m_{h}^{2}\right) .
$$

We defined $A_{1}^{\mu \nu}$ and $A_{2}^{\mu \nu}$ as

$$
\begin{aligned}
A_{1}^{\mu \nu}= & g^{\mu \nu}-\frac{p_{1}^{\nu} p_{2}^{\mu}}{\left(p_{1} \cdot p_{2}\right)}, \\
A_{2}^{\mu \nu}= & -g^{\mu \nu}+\frac{m_{h}^{2} p_{1}^{\nu} p_{2}^{\mu}}{p_{T}^{2}\left(p_{1} \cdot p_{2}\right)} \\
& -2 \frac{\left(p_{3} \cdot p_{2}\right) p_{1}^{\nu} p_{3}^{\mu}+\left(p_{3} \cdot p_{1}\right) p_{3}^{\nu} p_{2}^{\mu}-\left(p_{1} \cdot p_{2}\right) p_{3}^{\mu} p_{3}^{\nu}}{p_{T}^{2}\left(p_{1} \cdot p_{2}\right)},
\end{aligned}
$$

with $p_{T}$ the transverse momentum of the Higgs particle, which can be expressed in terms of the Mandelstam variables as

$$
p_{T}^{2}=\frac{\hat{t} \hat{u}-m_{h}^{4}}{\hat{s}} .
$$

The Born cross section, then, is

$$
\sigma^{(0)}(\hat{s})=\frac{G_{\mu} \alpha_{s}^{2}\left(\mu_{R}\right)}{512(2 \pi)^{3}} \int_{\hat{t}_{-}}^{\hat{t}_{+}} d \hat{t}\left(\left|T_{F} F_{1}\right|^{2}+\left|T_{F} F_{2}\right|^{2}\right),
$$

with $\hat{t}_{ \pm}=-\hat{s} / 2\left(1-2 m_{h}^{2} / \hat{s} \mp \sqrt{1-4 m_{h}^{2} / \hat{s}}\right)$. For our purpose, it is particularly convenient to introduce the prime Mandelstam variables:

$$
\begin{aligned}
s^{\prime}=p_{1} \cdot p_{2} & =\frac{\hat{s}}{2}, \quad t^{\prime}=p_{1} \cdot p_{3}=\frac{\hat{t}-m_{h}^{2}}{2}, \\
u^{\prime} & =p_{2} \cdot p_{3}=\frac{\hat{u}-m_{h}^{2}}{2},
\end{aligned}
$$

for which $s^{\prime}+t^{\prime}+u^{\prime}=0$. In these variables the Higgs transverse momentum becomes

$$
p_{T}^{2}=2 \frac{t^{\prime} u^{\prime}}{s^{\prime}}-m_{h}^{2}
$$

Our ultimate goal is to make an expansion for small $\sqrt{p_{T}^{2}+m_{h}^{2}} \sim p_{T}$. Since the final result is symmetrical in $t^{\prime} \leftrightarrow u^{\prime}$, the latter can be achieved expanding for $t^{\prime} \sim 0$, $u^{\prime} \sim-s^{\prime}$ (expanding only in $t^{\prime} \sim 0$ would not be correct if the final result were not symmetrical in $t^{\prime} \leftrightarrow u^{\prime}$ ). This is going to restrict $F_{1}$ and $F_{2}$ in Eq. (3) to a forward kinematic, namely to be a function of $\hat{s} / m_{t}^{2}$ only, reducing the computational difficulty from a three scales problem to a single scale one. To perform the expansion we need to express the momenta in terms of the parallel and transverse components with respect to the beam axis. For this purpose we define the combination of momenta:

$$
r=p_{1}+p_{3} \quad \text { and } \quad \bar{r}=p_{2}+p_{3} .
$$

It is easy to show that

$$
\begin{aligned}
r^{2}=\hat{t}, & \bar{r}^{2}=\hat{u}, \\
p_{1} \cdot r=-p_{2} \cdot r & =t^{\prime}, \quad p_{2} \cdot \bar{r}=-p_{1} \cdot \bar{r}=u^{\prime},
\end{aligned}
$$

and that

$$
r^{\mu}=\frac{t^{\prime}}{s^{\prime}}\left(-p_{1}^{\mu}+p_{2}^{\mu}\right)+r_{\perp}^{\mu}, \bar{r}^{\mu}=\frac{u^{\prime}}{s^{\prime}}\left(p_{1}^{\mu}-p_{2}^{\mu}\right)+\bar{r}_{\perp}^{\mu},
$$

where $r_{\perp}^{\mu}=\bar{r}_{\perp}^{\mu}$ is perpendicular to $p_{1}$ and $p_{2}$ and, as expected,

$$
r_{\perp}^{2}=m_{h}^{2}+2 t^{\prime}+2 \frac{t^{\prime 2}}{s^{\prime}}=-p_{T}^{2}
$$

Finally, in this reparametrization, $A_{1,2}^{\mu}$ assume particularly simple forms:

$$
A_{1}^{\mu \nu}=g^{\mu \nu}-\frac{p_{1}^{\nu} p_{2}^{\mu}}{s^{\prime}}, \quad A_{2}^{\mu \nu}=A_{1}^{\mu \nu}+2 \frac{r_{\perp}^{\mu} r_{\perp}^{\nu}}{p_{T}^{2}} .
$$

Expansion.-From Eq. (5), assuming real valued $\hat{t}$ and $\hat{u}$, we obtain the condition

$$
p_{T}^{2}+m_{h}^{2} \leq \frac{\hat{s}}{4},
$$

which allows us to expand for $p_{T}^{2} / s^{\prime} \ll 1$ and $m_{h}^{2} / s^{\prime} \ll 1$.

Although our program is clear, it is hindered by the fact that $p_{T}$ does not appear directly at the amplitude level. However, it is possible to show that an expansion for $r^{\mu} \sim 0^{\mu}$ is equivalent to an expansion in $p_{T}^{2} \sim 0$. Using Eqs. (11) and (12), and noticing that $r_{\perp}$ is purely spacelike, we can exchange the expansion in $p_{T}^{2} \sim 0$ with an expansion in $r^{\mu} \sim 0^{\mu}$ or, equivalently, $p_{3}^{\mu} \sim-p_{1}^{\mu}$. 
This observation is one of the main results of this Letter, and it allows us to proceed. We can then rewrite the form factors in Eq. (1) as

$$
\begin{aligned}
F_{1,2}= & \left.F_{1,2}\right|_{p_{3}=-p_{1}}+\left.r^{\mu} \frac{\partial F_{1,2}}{\partial p_{3}^{\mu}}\right|_{p_{3}=-p_{1}} \\
& +\left.\frac{1}{2} r^{\mu} r^{\nu} \frac{\partial^{2} F_{1,2}}{\partial p_{3}^{\mu} \partial p_{3}^{\nu}}\right|_{p_{3}=-p_{1}}+\cdots
\end{aligned}
$$

Although Eq. (14) is always valid, the expansion proposed in Eq. (15) requires a hierarchy between $r^{2}$ and $m_{t}^{2}$. We are going to estimate the range of validity of the small $p_{T}$ expansion by comparing at the LO the result obtained via Eq. (15) with the exact LO result (see next section).

We conclude this section with an important remark on how to correctly truncate the series in Eq. (15). Since the final result should be symmetrical for $p_{1} \leftrightarrow p_{2}$ and $\partial F_{1,2} / \partial p_{3}^{\mu}$ is a rank 1 tensor, the second term in the rhs of Eq. (15) can be rewritten as $r^{\mu} \mathcal{F}_{1,2}\left(p_{1}^{\mu}+p_{2}^{\mu}\right)=$ $\mathcal{F}_{1,2}(\hat{t}+\hat{u})$, with $\mathcal{F}_{1,2}$ a function of $s^{\prime}$ and $m_{t}^{2}$. For similar arguments, the third term should instead be proportional to $r^{\mu} r^{\nu}\left(g_{\mu \nu}+\cdots\right)=p_{T}^{2}+\cdots$. It is clear, then, that to expand to the first order in $p_{T}^{2}$, one has to expand to the second order in $p_{3}^{\mu}$, or, more in generally, an order $n$ expansion in $p_{T}^{2}$ needs the order $2 n$ expansion in $p_{3}^{\mu}$.

Computation and results.-We generated the relevant amplitudes for the virtual NLO corrections to $g g \rightarrow H H$ with FEYNARTS [37]. The amplitudes were contracted with the two orthogonal projectors in Eq. (4), using FEYNCALC [38], and reduced to a combination of scalar integrals. The integrals were then Taylor expanded, as described in the previous section. Subsequently, the resulting integrals were reduced in terms of a basis of master integrals using FIRE [39] and LITERED [40]. All of the master integrals, of which nearly the totality can be expressed in terms of multiple polylogarithms, were already known in the literature [41-47]. However, we evaluated them again directly in the phase space region of interest. We cross-checked our results using SECDEC [48]. The details of the calculation presented here, as well as a detailed study of the validity of our approximation at the hadronic level, will be the topic of a second paper on this argument [49], while in this Letter we will focus on the final result.

In order to show that our method correctly describes the partonic cross section for $\sqrt{\hat{s}}<750 \mathrm{GeV}$, we will start applying it to the LO.

In Fig. 1 we report our calculation for the partonic cross section using Eq. (15). As discussed in the Introduction, while the heavy $m_{t}$ expansion describes well only the range $\sqrt{\hat{s}}<2 m_{t}$, with our method we are able to correctly describe a wider range. It is also interesting to note that an expansion up to order $\mathcal{O}\left(p_{T}^{4}\right)$ is already sufficient to describe the complete result with enough precision.

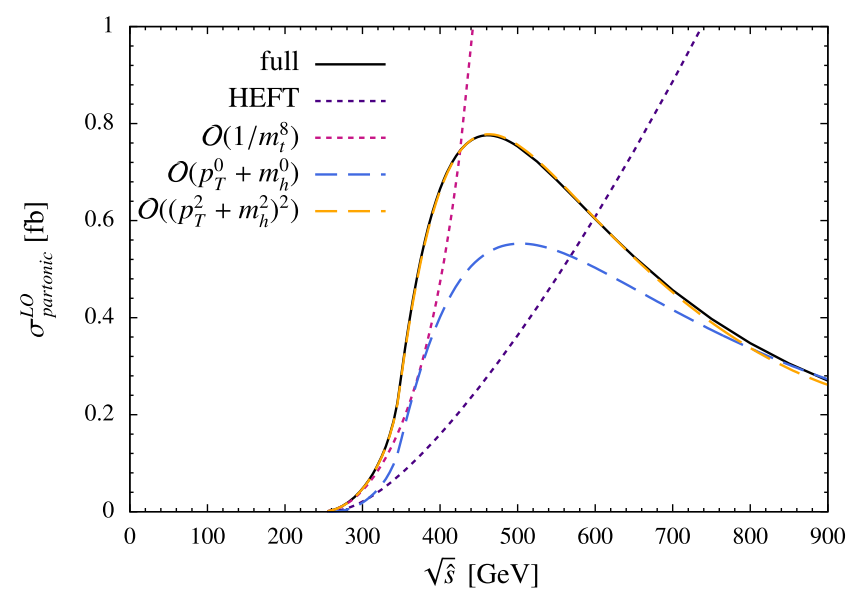

FIG. 1. Partonic cross section of $g g \rightarrow H H$ as a function of the partonic center-of-mass energy. The black continuous line is the full result [29]. The dotted lines represent the line of Higgs effective theory (HEFT) corresponding to the zero order in the heavy $m_{t}$ limit and the 4 th order in the heavy $m_{t}$ limit. The dashed lines are the result of the small $p_{T}^{2}$ approximation presented in this Letter.

The range of validity of the small $p_{T}$ expansion can be estimated comparing the partonic cross section calculated with our method with the one from the full LO calculation.

In Table I, we show

$$
\Delta \sigma=\left|\frac{2\left(\sigma_{\text {full }}-\sigma_{\text {approx }}\right)}{\left(\sigma_{\text {full }}+\sigma_{\text {approx }}\right)}\right|,
$$

where $\sigma_{\text {full }}$ is the cross section calculated without expansion, and $\sigma_{\text {approx }}$ is the one calculated in this Letter. Table I indicates that $\Delta \sigma$ is small and very well under control up to values of the partonic c.m. energy of about $\sim 750 \mathrm{GeV}$. Moreover, in the region of interest, the approximation rapidly improves as one considers higher order in the expansion in $p_{T}^{2}$ and $m_{h}^{2}$. The range of validity of our formulas is complementary to the one present in the literature, and represents $95 \%$ of the total hadronic cross section.

This behavior is confirmed (and even improved) in the comparison with the full numerical result at NLO. It is well known that the NLO virtual corrections are IR divergent and these divergences cancel against the ones that come

TABLE I. Relative difference between the approximated and the exact LO cross sections, for different orders of expansion, at various $\hat{s}$.

\begin{tabular}{lrrrrrr}
\hline \hline$\Delta \sigma-\hat{s}$ & $4 m_{t}^{2}$ & $6 m_{t}^{2}$ & $8 m_{t}^{2}$ & $12 m_{t}^{2}$ & $16 m_{t}^{2}$ & $32 m_{t}^{2}$ \\
\hline$p_{T}^{0} \times 10^{-1}$ & 6.2 & 4.4 & 3.2 & 1.8 & 1.0 & 0.3 \\
$p_{T}^{2} \times 10^{-2}$ & 8.5 & 4.4 & 1.1 & 2.4 & 5.1 & 33.2 \\
$p_{T}^{4} \times 10^{-2}$ & 1.3 & 0.1 & 0.4 & 0.2 & 0.9 & 2.8 \\
$p_{T}^{6} \times 10^{-3}$ & 2.3 & 0.9 & 1.0 & 0.1 & 3.5 & 450 \\
\hline \hline
\end{tabular}




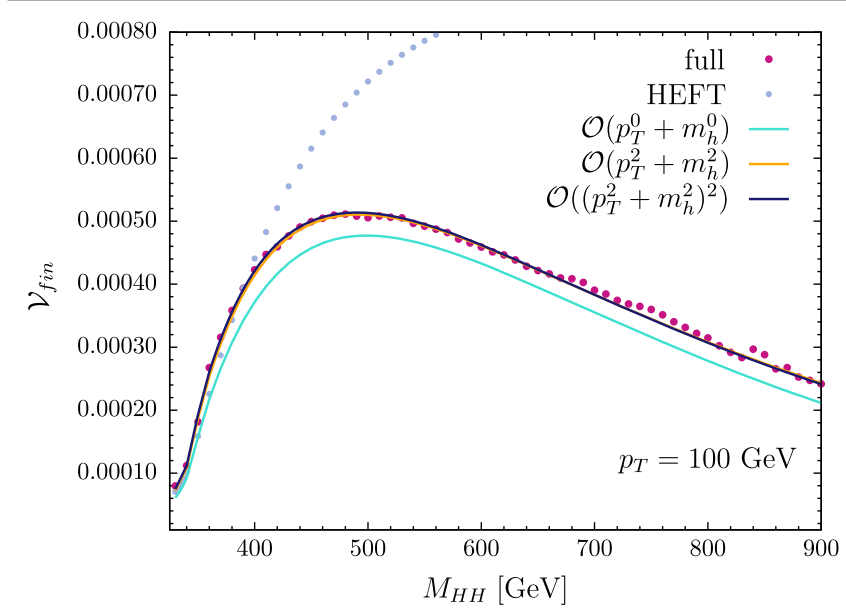

FIG. 2. Finite part of the virtual corrections as a function of the invariant mass of the two Higgs system. The pink points are extracted with the interpolation function from Ref. [50]. The dotted light blue points correspond to reweighted HEFT [51]. The solid lines are the respective orders in our calculation. We do not show $\mathcal{O}\left(\left(p_{T}^{2}+m_{h}^{2}\right)^{3}\right)$ as the line lies perfectly on top of the one of $\mathcal{O}\left(\left(p_{T}^{2}+m_{h}^{2}\right)^{2}\right)$.

from real corrections [32-34]. Following Ref. [34], we cancel the IR divergences by adding the counterterm $1 /\left(2 \epsilon^{2}\right) F_{1,2}^{\mathrm{LO}}\left(\epsilon^{2}\right)(\hat{s})^{-\epsilon}$, where $F_{1,2}^{\mathrm{LO}}(\epsilon)$ are the $\mathrm{LO}$ form factors with the inclusion of the $\mathcal{O}\left(\epsilon, \epsilon^{2}\right)$ terms. In Fig. 2 we compare our result to the numerical results from Ref. [30], at the partonic level, using the grid and the interpolation function for the finite part of the virtual corrections $\mathcal{V}_{\text {fin }}$ provided in Ref. [50]. As can be inferred from the figure, our expansion perfectly agrees with the full result when the first correction in $p_{T}$ and $m_{h}$ is included. It can clearly be seen that our lines smooth out the error on the full result stemming from the interpolation. Furthermore, we compare the numerical results of the authors of Ref. [30] with ours in several points of the grid provided by the same authors. For $p_{T} \lesssim 200 \mathrm{GeV}$, we find agreement between the two computations within the error quoted for each point of the grid from the numerical integration. For larger $p_{T}$ the agreement is still quite good (for $p_{T} \lesssim$ $300 \mathrm{GeV}$ is within twice the numerical error quoted), showing a degradation with the increase of $p_{T}$.

Conclusion.-In this Letter we have proposed a novel approach for the analytical computation of the NLO virtual corrections to Higgs pair production through gluon fusion. This method, based on an expansion for small $p_{T}^{2}$, allows us to describe accurately the region $\hat{s} \lesssim 750 \mathrm{GeV}$ that until now has been explored only numerically. In particular, we showed that a few terms in the expansion already reproduce the full LO within $10^{-3}$, in the region of interest. At NLO we find excellent agreement already at $\mathcal{O}\left(p_{T}^{2}+m_{h}^{2}\right)$ comparing to the full result of Ref. [30]. To judge the usefulness of our analytic method, we compare the CPU time needed to produce a phase-space point in our approach with that needed in the numerical calculation of Ref. [30]. In order to compute one single phase-space point, Ref. [30] quotes an average of $2 \mathrm{~h}$ per node using 16 Dual NVDIA TESLA K20X GPU nodes, while in our approach the computation of one single phase-space point took $4 \mathrm{sec}$ on a MacBook Air. We remark that this method is general and can be useful for the analytic computation of radiative corrections to other fundamental processes for the physics program of the LHC. In particular, the application of this method to the computation of the NLO virtual corrections to the top contribution in the $H Z, Z Z$, and $\gamma \gamma$ gluon fusion production processes is expected to be straightforward, while processes where the top and bottom contribution cannot be separate, like, e.g., in the $W W$ gluon fusion production, deserve a more detailed investigation.

P.P. G. would like thank Sally Dawson for insightful discussion. The work of P. P. G. is supported by the U.S. Department of Energy under Grant Contracts DESC0012704. R. G. would like to thank Stefan Jahn and Johannes Schlenk for help with SECDEC and the INFN, Sezione di Roma Tre and the theory group of LNF for their hospitality. R. G. is supported by a European Union COFUND/Durham Junior Research Fellowship under the EU Grant No. 609412.

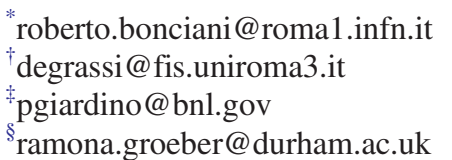

[1] T. Plehn and M. Rauch, The quartic Higgs coupling at hadron colliders, Phys. Rev. D 72, 053008 (2005).

[2] T. Binoth, S. Karg, N. Kauer, and R. Ruckl, Multi-Higgs boson production in the standard model and beyond, Phys. Rev. D 74, 113008 (2006).

[3] A. Djouadi, W. Kilian, M. Muhlleitner, and P. M. Zerwas, Production of neutral Higgs boson pairs at LHC, Eur. Phys. J. C 10, 45 (1999).

[4] U. Baur, T. Plehn, and D. L. Rainwater, Probing the Higgs self-coupling at hadron colliders using rare decays, Phys. Rev. D 69, 053004 (2004),

[5] J. Baglio, A. Djouadi, R. Gröber, M. M. Mühlleitner, J. Quevillon, and M. Spira, The measurement of the Higgs self-coupling at the LHC: Theoretical status, J. High Energy Phys. 04 (2013) 151.

[6] W. Yao, Studies of measuring Higgs self-coupling with $H H \rightarrow b \bar{b} \gamma \gamma$ at the future hadron colliders, in Proceedings of the 2013 Community Summer Study on the Future of U.S. Particle Physics: Snowmass on the Mississippi (CSS2013): Minneapolis, MN, 2013, arXiv:1308.6302.

[7] V. Barger, L. L. Everett, C. B. Jackson, and G. Shaughnessy, Higgs-pair production and measurement of the triscalar coupling at LHC(8,14), Phys. Lett. B 728, 433 (2014).

[8] A. Azatov, R. Contino, G. Panico, and M. Son, Effective field theory analysis of double Higgs boson production via gluon fusion, Phys. Rev. D 92, 035001 (2015). 
[9] C.-T. Lu, J. Chang, K. Cheung, and J. S. Lee, An exploratory study of Higgs-boson pair production, J. High Energy Phys. 08 (2015) 133.

[10] M. J. Dolan, C. Englert, and M. Spannowsky, Higgs selfcoupling measurements at the LHC, J. High Energy Phys. 10 (2012) 112.

[11] A. Papaefstathiou, L. L. Yang, and J. Zurita, Higgs boson pair production at the LHC in the $b \bar{b} W^{+} W^{-}$channel, Phys. Rev. D 87, 011301 (2013).

[12] D. E. F. de Lima, A. Papaefstathiou, and M. Spannowsky, Standard model Higgs boson pair production in the $(b \bar{b})(b \bar{b})$ final state, J. High Energy Phys. 08 (2014) 030.

[13] D. Wardrope, E. Jansen, N. Konstantinidis, B. Cooper, R. Falla, and N. Norjoharuddeen, Non-resonant Higgs-pair production in the $b \bar{b} b \bar{b}$ final state at the LHC, Eur. Phys. J. C 75, 219 (2015).

[14] J. K. Behr, D. Bortoletto, J. A. Frost, N. P. Hartland, C. Issever, and J. Rojo, Boosting Higgs pair production in the $b \bar{b} b \bar{b}$ final state with multivariate techniques, Eur. Phys. J. C 76, 386 (2016).

[15] M. J. Dolan, C. Englert, N. Greiner, and M. Spannowsky, Further on Up the Road: $h h j j$ Production at the LHC, Phys. Rev. Lett. 112, 101802 (2014).

[16] M. J. Dolan, C. Englert, N. Greiner, K. Nordstrom, and M. Spannowsky, hhjj production at the LHC, Eur. Phys. J. C 75, 387 (2015).

[17] C. Englert, F. Krauss, M. Spannowsky, and J. Thompson, Di-Higgs phenomenology in $t \bar{t} h h$ : The forgotten channel, Phys. Lett. B 743, 93 (2015).

[18] S. Dawson, A. Ismail, and I. Low, What's in the loop? The anatomy of double Higgs production, Phys. Rev. D 91, 115008 (2015).

[19] C. O. Dib, R. Rosenfeld, and A. Zerwekh, Double Higgs production and quadratic divergence cancellation in little Higgs models with T parity, J. High Energy Phys. 05 (2006) 074.

[20] R. Gröber and M. Mühlleitner, Composite Higgs boson pair production at the LHC, J. High Energy Phys. 06 (2011) 020.

[21] R. Contino, M. Ghezzi, M. Moretti, G. Panico, F. Piccinini, and A. Wulzer, Anomalous couplings in double Higgs production, J. High Energy Phys. 08 (2012) 154.

[22] R. Gröber, M. Mühlleitner, and M. Spira, Signs of composite Higgs pair production at next-to-leading order, J. High Energy Phys. 06 (2016) 080.

[23] G. Aad et al. (ATLAS Collaboration), Search For Higgs Boson Pair Production in the $\gamma \gamma b \bar{b}$ Final State Using $p p$ Collision Data at $\sqrt{s}=8 \mathrm{TeV}$ from the ATLAS Detector, Phys. Rev. Lett. 114, 081802 (2015).

[24] V. Khachatryan et al. (CMS Collaboration), Search for resonant pair production of Higgs bosons decaying to two bottom quark-antiquark pairs in proton-proton collisions at $8 \mathrm{TeV}$, Phys. Lett. B 749, 560 (2015).

[25] G. Aad et al. (ATLAS Collaboration), Search for Higgs boson pair production in the $b \bar{b} b \bar{b}$ final state from $p p$ collisions at $\sqrt{s}=8 \mathrm{TeV}$ with the ATLAS detector, Eur. Phys. J. C 75, 412 (2015).

[26] G. Aad et al. (ATLAS Collaboration), Searches for Higgs boson pair production in the $h h \rightarrow b b \tau \tau, \gamma \gamma W W^{*}, \gamma \gamma b b$, $b b b b$ channels with the ATLAS detector, Phys. Rev. D 92, 092004 (2015).
[27] V. Khachatryan et al. (CMS Collaboration), Search for two Higgs bosons in final states containing two photons and two bottom quarks in proton-proton collisions at $8 \mathrm{TeV}$, Phys. Rev. D 94, 052012 (2016).

[28] M. Aaboud et al. (ATLAS Collaboration), Search for pair production of Higgs bosons in the $b \bar{b} b \bar{b}$ final state using proton-proton collisions at $\sqrt{s}=13 \mathrm{TeV}$ with the ATLAS detector, Phys. Rev. D 94, 052002 (2016).

[29] E. W. N. Glover and J. J. van der Bij, Higgs boson pair production via gluon fusion, Nucl. Phys. B309, 282 (1988).

[30] S. Borowka, N. Greiner, G. Heinrich, S. Jones, M. Kerner, J. Schlenk, U. Schubert, and T. Zirke, Higgs Boson Pair Production in Gluon Fusion at Next-to-Leading Order with Full Top-Quark Mass Dependence, Phys. Rev. Lett. 117, 012001 (2016); Erratum: Higgs Boson Pair Production in Gluon Fusion at Next-to-Leading Order with Full TopQuark Mass Dependence, Phys. Rev. Lett. 117, 012001 (2016); Erratum, Phys. Rev. Lett.117, 079901(E) (2016).

[31] S. Borowka, N. Greiner, G. Heinrich, S. P. Jones, M. Kerner, J. Schlenk, and T. Zirke, Full top quark mass dependence in Higgs boson pair production at NLO, J. High Energy Phys. 10 (2016) 107.

[32] J. Grigo, J. Hoff, K. Melnikov, and M. Steinhauser, On the Higgs boson pair production at the LHC, Nucl. Phys. B875, 1 (2013).

[33] J. Grigo, J. Hoff, and M. Steinhauser, Higgs boson pair production: Top quark mass effects at NLO and NNLO, Nucl. Phys. B900, 412 (2015).

[34] G. Degrassi, P. P. Giardino, and R. Gröber, On the two-loop virtual QCD corrections to Higgs boson pair production in the standard model, Eur. Phys. J. C 76, 411 (2016).

[35] J. Davies, G. Mishima, M. Steinhauser, and D. Wellmann, Double-Higgs boson production in the high-energy limit: Planar master integrals, J. High Energy Phys. 03 (2018) 048.

[36] R. Gröber, A. Maier, and T. Rauh, Reconstruction of topquark mass effects in Higgs pair production and other gluonfusion processes, J. High Energy Phys. 03 (2018) 020.

[37] T. Hahn, Generating Feynman diagrams and amplitudes with FeynArts 3, Comput. Phys. Commun. 140, 418 (2001).

[38] V. Shtabovenko, R. Mertig, and F. Orellana, New developments in FeynCalc 9.0, Comput. Phys. Commun. 207, 432 (2016).

[39] A. V. Smirnov, FIRE5: A C++ implementation of Feynman Integral REduction, Comput. Phys. Commun. 189, 182 (2015).

[40] R. N. Lee, LiteRed 1.4: A powerful tool for reduction of multiloop integrals, J. Phys. Conf. Ser. 523, 012059 (2014).

[41] R. Bonciani, P. Mastrolia, and E. Remiddi, Vertex diagrams for the QED form-factors at the 2-loop level, Nucl. Phys. B661, 289 (2003); Erratum to: Vertex diagrams for the QED form factors at the 2-loop level, Nucl. Phys. B661, 289 (2003); Erratum, Nucl. Phys.B702, 359(E) (2004).

[42] R. Bonciani, P. Mastrolia, and E. Remiddi, Master integrals for the two loop QCD virtual corrections to the forward backward asymmetry, Nucl. Phys. B690, 138 (2004).

[43] U. Aglietti, R. Bonciani, G. Degrassi, and A. Vicini, Analytic results for virtual QCD corrections to Higgs production and decay, J. High Energy Phys. 01 (2007) 021. 
[44] C. Anastasiou, S. Beerli, S. Bucherer, A. Daleo, and Z. Kunszt, Two-loop amplitudes and master integrals for the production of a Higgs boson via a massive quark and a scalar-quark loop, J. High Energy Phys. 01 (2007) 082.

[45] M. Becchetti and R. Bonciani, Two-Loop master integrals for the planar QCD massive corrections to di-photon and di-jet hadro-production, J. High Energy Phys. 01 (2018) 048.

[46] S. Caron-Huot and J. M. Henn, Iterative structure of finite loop integrals, J. High Energy Phys. 06 (2014) 114.

[47] A. von Manteuffel and L. Tancredi, A non-planar two-loop three-point function beyond multiple polylogarithms, J. High Energy Phys. 06 (2017) 127.
[48] S. Borowka, G. Heinrich, S. P. Jones, M. Kerner, J. Schlenk, and T. Zirke, SecDec-3.0: Numerical evaluation of multi-scale integrals beyond one loop, Comput. Phys. Commun. 196, 470 (2015).

[49] R. Bonciani, G. Degrassi, P. P. Giardino, and R. Gröber (to be published).

[50] G. Heinrich, S. P. Jones, M. Kerner, G. Luisoni, and E. Vryonidou, NLO predictions for Higgs boson pair production with full top quark mass dependence matched to parton showers, J. High Energy Phys. 08 (2017) 088.

[51] S. Dawson, S. Dittmaier, and M. Spira, Neutral Higgs boson pair production at hadron colliders: QCD corrections, Phys. Rev. D 58, 115012 (1998). 